Recepción: 22/08/2018

Evaluación: 22/ 08/2018

Aprobación: 26/10/2018

Artículo de Investigación-Reflexión

DOI: https://doi.org/10.22267/rhec.192222.55

\title{
El modelo Tyleriano de curriculum y el papel de los objetivos conductuales. ¿Tyler fue realmente conductista?
}

\author{
José María García Garduño ${ }^{1}$ \\ Universidad Autónoma de la Ciudad de México, México
}

\section{Resumen}

El propósito de este trabajo de investigación es clarificar algunas omisiones y distorsiones acerca de la obra de Tyler desde finales de la década de los 70, sobre todo el papel de los objetivos conductuales en el currículo, así como presentar algunos aspectos de su vida que no son muy conocidos en Iberoamérica. Parte de la información que se emplea en este trabajo se basa en diversas fuentes bibliográficas y entrevistas que el autor dio a lo largo de su vida. Se concluye que aunque Tyler empleó los objetivos conductuales, fue un crítico de los mismos. Asimismo, el pensamiento tyleriano nunca fue estático. Al final de su vida el pensamiento del autor tenía ciertos tintes constructivistas.

Palabras clave: diseño curricular; historia de la educación; historia del currículo; teoría curricular; objetivos conductuales.

Doctor en Administración Educativa por la Ohio University, Estados Unidos. Grupo de Investigación: Didáctica UNAM, México. Línea de investigación: Evaluación y Gestión Educativa. Correo electrónico: josemariagarduno@gmail.com. Código ORCID: https://orcid. org/0000-0002-0955-0880 


\title{
The Tylerian curriculum model and the role of the behavioral objectives ¿Was Tyler a real behaviorist?
}

\begin{abstract}
The purpose of this research work is to clarify some omissions and distortions about Tyler's work since the late 1970s, especially the role of behavioral objectives in the curriculum, as well as to present some aspects of his life that are not well known in Ibero-America. Part of the information used in this work is based on several bibliographic sources and interviews that the author gave throughout his life. It is concluded that although Tyler used behavioral objectives, he was critical of them. Likewise, Tylerian thought was never static. At the end of his life the author's thought had certain constructivism hints.
\end{abstract}

Keywords: Curricular Design; Education History; Curriculum History; Curricular Theory; Behavioral Objectives.

\section{O modelo curricular Tyleriano e o papel dos objetivos comportamentais ¿Tyler era realmente comportamental?}

\section{Resumo}

O objetivo deste trabalho de pesquisa é esclarecer algumas omissões e distorções sobre o trabalho de Tyler desde o final da década de 1970, especialmente o papel dos objetivos comportamentais no currículo, além de apresentar alguns aspectos de sua vida que não são muito conhecidos na América Latina. Algumas das informações utilizadas neste trabalho são baseadas em várias fontes bibliográficas e entrevistas que o autor deu ao longo de sua vida. Conclui-se que, embora Tyler empregou objetivos comportamentais, ele foi um crítico deles. Da mesma forma, o pensamento Tyleriano nunca foi estático. No final de sua vida, o pensamento do autor apresentava certos corantes construtivistas.

Palabras-chave: desenho curricular; história da educação; histórico curricular; teoria curricular; objetivos comportamentais. 


\section{Introducción}

"En tanto exista educación habrá curriculum" Ralph W. Tyler (1990)

El propósito de este trabajo es clarificar algunas omisiones y distorsiones acerca de la obra de Tyler desde finales de la década de los 70, sobre todo el papel de los objetivos conductuales en el currículo, así como presentar algunos aspectos de su biografía, poco conocidos en Iberoamérica. Parte de la información que se emplea en este trabajo se basa en diversas fuentes bibliográficas y entrevistas que el autor dio a lo largo de su vida; especialmente, en la entrevista que el autor concedió a Graciela Cordero, en 1990 (Cordero y García Garduño, 2004). Esa entrevista fue la primera que Ralph Tyler dio a un especialista del medio iberoamericano.

Ralph Winfred Tyler nació en Chicago, el 22 de abril de 1902, y creció en Nebraska. Obtuvo el bachelor en 1921, del Doane College, con especialidad en ciencias y matemáticas. A los 20 años, en 1922, se convirtió en profesor de educación secundaria en la ciudad de Pierre, South Dakota. A los 21 años (1923), obtuvo su maestría de la Universidad de Nebraska, donde comenzó a especializarse en el uso de la estadística en las pruebas de rendimiento. En 1927 - tenía 25 años- obtuvo el doctorado en psicología educativa en la Universidad de Chicago. Por invitación de su profesor del doctorado, Werret Charters, Tyler se trasladó a Columbus para dirigir el Departamento de evaluación educativa, en la Ohio State University. Su obra y figura se volvió conocida en el medio educativo a partir del Estudio de los Ocho Años, la investigación curricular más grande que se hubiera emprendido hasta ahora (Cordero y García Garduño, 2004).

Tyler fue un hombre incansable. Desde 1921, cuando se graduó como maestro de escuela secundaria, hasta pocos meses antes de su muerte (1992), estuvo activo; es decir, unos 70 años. Se cuenta que Benjamin Bloom una vez hizo cuentas de cuánto tiempo Tyler había pasado viajando en avión y calculó que habían sido dos años completos de su vida. Una vez dijo, como si tratara de dejar una adicción, "trato de disminuir los viajes a una vez a la semana" (Husen, 1989, viii).

A Ralph W. Tyler se lo considera uno de los educadores más importantes del siglo XX en Estados Unidos; fue docente, investigador, consultor y funcionario. Tyler ocupó varios puestos y fundó varios centros de investigación. Fue 
asesor de varios presidentes de los Estados Unidos, trabajó en varios Comités y Consejos nacionales. Fue, también, miembro de la Comisión Nacional para la Formación de profesores (National Comisión on Teacher Education), de 1939 a 1946. Perteneció al Nacional Science Board, de 1962 a 1968. En palabras de uno de sus hijos, Tyler rechazaba idealizar el pasado y denigrar el presente, una tentación a la que sucumbieron varios de sus colegas (Stanford, News Service, 1994).

A este gran educador e investigador se le atribuye, entre otras cosas, el haber fundado el campo de la evaluación educativa; fue el creador del primer método para construir pruebas de rendimiento y haber creado el modelo de desarrollo curricular más empleado: el método tyleriano de construcción del curriculum (Tyler's rationale). Entre sus estudiantes, destacan Benjamín Bloom y Lee J. Cronbach (1916-2001), calificados ambos, por Tyler, como su brazo derecho (Schubert y López Schubert, 1986).

Bloom (1986), por ejemplo, manifiesta que él venía de una familia de clase trabajadora y que Tyler le tuvo confianza e impulsó su carrera. Según Bloom, Tyler era un gurú. Afirmaba que cuando "uno escuchaba lo que decía, aun cuando no entendieras, el problema era encontrarle un significado más profundo" (Bloom, 1986: 36). Ahora, entonces, se puede entender por qué el libro de Bloom (1971), Taxonomía de los objetivos de la educación, lo dedicó a su antiguo maestro.

Ralph Tyler fue un hombre virtuoso y modesto. John Goodlad, otro de sus discípulos destacados, señala:

Tal vez su contribución más grande sea el consejo que dio a cientos de individuos, en forma personal. En una combinación de rasgos hace que media hora con él sea tan recompensable: la habilidad para concentrarse exclusivamente en el problema que está ante él y la habilidad para plantear las preguntas correctas. Maestro, académico, administrador, creador de instituciones, hacedor de políticas, orador, viajero, asesor de presidentes, consejero, amigo - Ralph Tyler ha sido todo eso y en todos estos papeles que jugó, siempre fue un estudiante (Schubert y López Schubert, 1986a: 10-11).

En una entrevista que le concedió (Ridings, 1981), el entrevistador le manifestó a Tyler que lo consideraba como un gurú de la educación, como una de las figuras más importantes en la educación estadounidense. Tyler le respondió que los halagos no conducían a ninguna parte, que mejor continuaran con la entrevista. Tal vez la modestia natural que tuvo Tyler provino de su familia. Este 
autor le manifestó a una de sus biógrafos (Meek, 1993) que su padre fue médico de profesión, que a finales del siglo XIX ganaba unos cinco mil dólares —una cantidad muy respetable en esa época-, pero que se retiró de la profesión para convertirse en pastor de una iglesia. A su padre no le importó que ganara menos como ministro de la iglesia. Probablemente por el ejemplo de su padre, Tyler abrazó el humanismo. En una entrevista, Tyler declaró que la vida plena de un ser humano es aquella en la cual ese ser humano está buscando continuamente ser más humano:

Desde mi punto de vista una buena vida para un ser humano es una en la cual está buscando ser un humano más pleno; lo que significa ser más capaz de aprender, de ayudar a otros; de contribuir; de crear una sociedad en la cual otros tengan un profundo respeto por el potencial de cada ser humano y de estar fuertemente motivado a buscar ser parte de esa sociedad que estaría haciendo mejores cosas para las generaciones futuras (Schubert et al., 1986b: 100).

\section{Tyler y los objetivos conductuales en el currículo}

En el medio iberoamericano se conoce a Tyler principalmente por su obra Principios básicos del currículo (1973/1949), trabajo que posiblemente ha sido la obra que más influencia ha tenido en la constitución y práctica del curriculum a nivel mundial. Sin embargo, su pensamiento se lo ha distorsionado frecuentemente y, en el reconocimiento de sus aportaciones, se registran algunas omisiones. Se considera, por ejemplo, que Tyler era conductista, por su énfasis en el uso de objetivos conductuales en la construcción y evaluación del curriculum.

Los pioneros de los objetivos conductuales fueron dos profesores de la Universidad de Chicago, Werret W. Charters (1923) y J. Franklin Bobbitt (1924). Estos autores diseñaban programas con cientos e incluso miles de objetivos. Por ejemplo, en la obra cumbre de Bobbitt (1924), How to make a curriculum, sugiere más de 800 objetivos para la educación básica y secundaria. Bobbitt, en esta misma obra, señala que se deben evitar los objetivos vagos y generales. A pesar de que Tyler fue estudiante y después colaborador de Charters, no se ciñó del todo a este enfoque de objetivos.

Tyler, en la obra escrita originalmente en 1949 (Tyler, 1973: 47), señala que: "como resultado de los pasos precedentes, el planificador del currículo habrá podido seleccionar una pequeña lista de objetivos importantes y alcanzables que, 
por provenir de varias fuentes, podrán enunciarse de distintas maneras". Si bien Tyler aceptaba, como Bobbit y Charters, que los objetivos debían establecerse en términos de conducta, su pensamiento se ligaba más a Dewey y tuvo, también, influencia de su profesor y director de tesis doctoral, Charles Judd (1873-1946), de quien retomó la importancia de la generalización en el currículo y los objetivos (Cordero y García Garduño, 2004), así como que unas de las fuentes del curriculum fueran la sociedad y los estudios de los estudiantes (Tyler, 1943. "Charles Hubbard Judd, 1873-1846”. The Elementary School Journal, 47(1):1-2)

Tyler tuvo como maestra, en la Universidad de Nebraska, a una discípula de Thorndike y, a través de ella, conoció su libro Psicología de la aritmética, que tenía 3000 objetivos; desde esa época, Tyler pensaba que los objetivos conductuales eran demasiado específicos (Schubert et al., 1986b).

En la década de los treinta, Tyler se refiere a objetivos transcurriculares (transcurriculum objectives), los que concebía como objetivos muy amplios, que debían atender el conjunto de la escuela o la comunidad (Cronbach, 1986). Un ejemplo de estos objetivos era desarrollar una filosofía de vida en los educandos.

Esta misma idea la expresó Tyler cuarenta años después, en pleno apogeo del uso de los objetivos conductuales. Tyler puntualizó algunas limitaciones de los objetivos conductuales; señaló que los objetivos eran específicos, pero no lo suficientemente claros (Fishbien, 1973). Posteriormente, señaló que: no se pueden usar los objetivos como base para una evaluación comprehensiva (Ridings, 1981, 12). En esa misma entrevista, Tyler destacó las limitaciones de las taxonomías educativas: "una taxonomía es lo que alguien más establece como un significado de los objetivos educativos, pero el profesor es quien trabaja con los alumnos" (Ridings, 1981, 13). Tyler mencionó que el profesor que trabaja con los alumnos debe establecer lo que es importante de aprender.

En la entrevista concedida a Graciela Cordero, en 1990 (Cordero y García Garduño, 2004), Tyler ilustra cómo evolucionó su pensamiento en torno a los objetivos. El autor señaló a la entrevistadora:

GC: ¿Cuál es su opinión sobre el establecimiento de objetivos conductuales en el curriculum?

Ralph Tyler: Los objetivos deben ser lo suficientemente amplios para que sean comprensibles. Las habilidades del ser humano son para generalizar, por lo tanto, 
cuando tienes algo específico no ayuda a generalizar el principio subyacente como algo nuevo. De otra manera se vuelve como una capacitación para que las personas hagan el trabajo, cosas pequeñas que ellas generalmente no entienden. Por lo tanto, no escojas objetivos conductuales que sean tan pequeños que no exista generalización. Eso no es humano, los seres humanos generalizan a partir de su experiencia. Por ejemplo, en la enseñanza de la lectura, uno de los objetivos es comprender. Otro puede ser desarrollar el interés en los materiales de lectura. Eso es un objetivo general, puedes ver varias posibilidades, pero no los lleves a cuestiones concretas (Cordero y García Garduño, 2004, 11-12).

Es decir, Tyler consideraba que los objetivos debían ser lo suficientemente amplios como para que el estudiante pueda generalizarlos. Sin embargo, Tyler tuvo una influencia de los curricularistas conductistas de su época: Franklin Bobbitt y Werret W. Charters; ambos fueron sus profesores en la Universidad de Chicago (García Garduño, 1995).

Bobbitt, junto con Charters, fue el pionero de los objetivos conductuales. Aquel autor (Bobbitt, 1924) señalaba que los objetivos generales deben evitarse y redactarse en términos definitivos, pues, de esa manera, los educadores saben qué es lo deben lograr y, también, es útil para que los padres y estudiantes entiendan tales objetivos. Asimismo, Bobbitt sugería que los objetivos se redactaran con un lenguaje claro y sencillo.

El modelo tyleriano del curriculum tuvo influencia del pensamiento de Charters, quien fue el creador del método de análisis de tareas (job analysis). Los pasos básicos de método para la elaboración del currículo, que Charters (1923) propuso, son:

1) determinar los objetivos más importantes de la educación;

2) analizar los objetivos y continuar su examen hasta el nivel de unidades de trabajo;

3) jerarquizar las actividades de acuerdo con su importancia;

4) seleccionar los objetivos y actividades que puedan ser alcanzados dentro del calendario escolar;

5) recolectar las mejores experiencias derivadas de las actividades seleccionadas;

6) ordenar los materiales de instrucción de acuerdo con la naturaleza psicológica del educando. 
Asímismo, en el medio iberoamericano, no se sabe mucho que la obra cumbre de Principios básicos del currículo (Tyler, 1973) no se pensó originalmente como un libro. Eran los apuntes de los cursos Education 305 y 360, que impartía en la Universidad de Chicago. Por esa razón, la obra no tiene referencias; se dice que la bibliografía de la edición en español la agregó el traductor de la obra. Tyler le manifestó a Graciela Cordero que eran apuntes de clase, que no pensaba publicar; que la Universidad de Chicago los publicó sin su conocimiento.

Las escasas 100 páginas de sus apuntes de clase fueron la piedra de toque para el desarrollo de la disciplina y práctica del curriculum como se conoce ahora.

Este libro fue producto del Estudio de los Ocho Años (1933-1941), una investigación longitudinal realizada en la época de depresión económica, cuyo propósito fue evaluar los efectos de la educación progresiva de Dewey en los estudios universitarios a través de una comparación con la educación tradicional. La Asociación para la Educación Progresista se encargó del estudio e incluyó 30 escuelas de educación media superior y 300 colegios universitarios (Tyler, 1986).

Algunos investigadores que participaban en las Comisiones le hicieron notar a Tyler que el Estudio contaba con un modelo de evaluación, pero no de curriculum. Tyler, quien se encontraba cenando con Hilda Taba, tomó una servilleta y ahí esquematizó lo que se conocería como el modelo tyleriano de curriculum (Ridings, 1981).

Si bien, ya en el año 1932, Tyler tenía elaborada gran parte del pensamiento que caracterizó su obra (Cronbach, 1986), no se mantuvo estático; evolucionó durante su larga y productiva carrera. Prácticamente, estuvo activo hasta sus últimos días de existencia. A los noventa años, dijo, en una ocasión: "no conozco a nadie que no pueda aprender" (Universidad de Stanford, News Service, 1994).

Este hombre extraordinario, que comenzó siendo conductista y deweyniano, terminó por abrazar, de alguna manera, el pensamiento constructivista, como podrá verse en la entrevista que Tyler le concedió a Cordero, en 1990. En esa entrevista, el autor expresó ideas que se pueden clasificar dentro del pensamiento constructivista. Por ejemplo, él consideraba que: 
Los profesores deben entender que la buena enseñanza no es seguir recetas sino comprender a los estudiantes y ayudarles a aprender. Los profesores pueden tener recetas para enseñar, pero eso no ayuda a los niños a aprender. Es más fácil enseñar a una persona a enseñar si le importan los alumnos que a alguien que no le importan y piensa que todo lo que él o ella necesita son métodos de enseñanza... La relación docente estudiante es más importante que todo el conocimiento que tienes acerca de la enseñanza, que efectivamente le respondas al alumno. ¿No lo crees así? (Cordero y García Garduño, 2004, 13)

Respecto al papel que el maestro debe tener en la docencia, sería difícil catalogarlo como conductista. Tyler relega el papel de los métodos de enseñanza y los subordina al papel del docente como mediador del aprendizaje; no concibe un buen docente que no tenga interés por sus alumnos.

Otras ideas de Tyler, que nos llevan a suponer los tintes constructivistas en su pensamiento educativo, son: "No se puede mejorar la educación de arriba hacia abajo... Uno tiene que ayudar a que la gente haga mejor trabajo" (Cordero y García Garduño, 2004, p. 14). Nuevamente, se puede observar la evolución del pensamiento tyleriano; concibe que las reformas educativas deben partir desde abajo; es decir, los maestros deben ser sus promotores.

En conclusión, puede afirmarse que la obra de Tyler fue crucial en el desarrollo del curriculum y en la evaluación; que su pensamiento evolucionó a lo largo de su longeva vida. Por otro lado, el autor empleaba de manera limitada el uso de los objetivos conductuales en el diseño del curriculum. Tyler, desde la década de los 30, se refirió a objetivos transcurriculares. Varias de sus ideas más recientes, expresadas en la entrevista que le concedió a Cordero, podrían considerarse dentro del pensamiento constructivista. 


\section{Referencias}

Bloom, Benjamin. Taxonomía de los objetivos de la educación: la clasificación de las metas educacionales. Buenos Aires: Centro Regional de Ayuda Técnica, 1971.

Bloom, Benjamin. "Ralph Tyler's impact on evaluation theory and practice". Journal of Thought. No. 21 (1986): 36-46.

Bobbitt, Franklin. How to make a curriculum. Boston/New York: Houghton Mifflin, Co., 1924.

Charters, Werret Wallace. Curriculum construction. Nueva York: Macmillan Co., 1923. (Reedición: New York: Arno Press, 1971).

Cordero Arroyo, Graciela, y García Garduño, José María E. (2004). “Tyler’s curriculum rationale and the reconceptualists. Interview with Ralph W. Tyler (1902-1994)". Revista Electrónica de Investigación Educativa, vol. 6. No. 2. http://redie.uabc.mx/vol6no2/contenido-cordero.html

Cronbach, Lee Joseph. "Tyler's contributions of the Eight Year Study". Journal of Thought. No. 21 (1986): 47-52.

García Garduño José María E. "La consolidación de la teoría curricular en los Estados Unidos (1912-1949)". Revista Latinoamericana de Estudios Educativos, vol. 25. No. 2 (1995): 57-81.

Fishbein, Justin M. "The father of behavioral objectives criticizes them: An interview with Ralph Tyler”. Phi Delta Kappan, vol. 55. No. 1 (1973): 55-57.

Meek, Anne. "On Setting the Highest Standards: A Conversation with Ralph Tyler". Educational Leadership, vol. 50. No. 6 (1993):83-86.

Ridings Nowakovski, Jeri. "An interview with Ralph Tyler". Journal of MultiDisciplinary Evaluation, vol. 7. No. 15 (2011): 202-226. http://journals. sfu.ca/jmde/index.php/jmde_1/article/view/304/299 
Schubert, William H., y Lopez Schubert, Ann Lynn. (1986a). "Ralph W. Tyler. An interview and antecedent reflections". Journal of Thought, vol. 21. No. 1 (1986a): 7-14.

Schubert, William H. et al. "A Dialogue with Ralph W. Tyler". Journal of Thought, vol. 21. No. 1 (1986b): 91-118.

Tyler, Ralph W. "Charles Hubbard Judd 1873-1846". The Elementary School Journal, vol. 47. No. 1 (1946): 1-2.

Tyler. Ralph W. (1973). Principios básicos del currículo. Buenos Aires: Troquel, 1973.

Tyler, Ralph W. (1986). "Reflecting on the Eight-Year Study". Journal of Thought, vol. 21. No. 1 (1986): 15-23.

Universidad de Stanford. "Ralph Tyler, one of century's foremost educators dies at 91". News Service (28 de febrero de 1994). www.stanford.edu/dept/ news/pr/94/940228Arc4425.html 